\title{
Genetic analyses of the major and minor locus groups of bacterial wilt resistance in tobacco using a diallel cross design
}

Y.L. Qian ${ }^{1 *}$, J. Chen ${ }^{2 *}$, J.J. Dong ${ }^{3}$, Z.C. Wu ${ }^{4}$, Y.H. Liu ${ }^{3}$, B.Y. Xue ${ }^{3}$, F.W. Shao ${ }^{3}$ and X.Y. Sun ${ }^{1}$

${ }^{1}$ Tobacco Research Institute, Anhui Academy of Agricultural Science, Hefei, China ${ }^{2}$ Institute of Agricultural Economy and Information, Anhui Academy of Agricultural Science, Hefei, China

${ }^{3}$ Anhui Provincial Corporation of China National Tobacco Corporation, Hefei, China ${ }^{4}$ Institute of Crop Science, Chinese Academy of Agricultural Sciences, Beijing, China

*These authors contributed equally to this study.

Corresponding author: X.Y. Sun

E-mail: sxyyxscn@yahoo.com

Genet. Mol. Res. 15 (1): gmr.15017223

Received July 15, 2015

Accepted November 9, 2015

Published January 29, 2016

DOI http://dx.doi.org/10.4238/gmr.15017223

\begin{abstract}
Tobacco germplasm samples with various levels of resistance to bacterial wilt were selected to construct $F_{1}$ combinations of parental inbred lines and orthogonal diallel crosses using samples collected in 2009 (15 germplasms), 2010 (15 germplasms), and 2011 (16 germplasms). A total of $1 / 2 P(P+1)$ experimental materials were used for analysis. Based on the analyses of major and minor locus groups, genetic effects on the incidence rate and index of bacterial wilt in tobacco were investigated on the 15th and 25th day during the early stage. Significant effects were observed in major locus groups, but not in minor locus groups. Specifically, adjacent major locus groups $(\mathrm{J} 1=13,056$ and $\mathrm{J} 1=13,055 ; \mathrm{J} 1=14,080$ and $\mathrm{J} 1=14,079$ ) were detected in both the first and second analyses with considerable effects. Based on the additive effects of minor locus groups on the rate and index of bacterial wilt, the effects on the incidence rates of
\end{abstract}


Yunyan 85, DB101, and RG11 as well as the effects on the disease index of the latter two germplasms reached the maximum. This was consistent with the disease resistance indicators of these tobacco varieties in the field (corresponding broad heritability $>20 \%$ ). Genetic homozygous dominant loci $(++)$ increased the rate of bacterial wilt (susceptible), whereas homozygous recessive loci (- -) reduced the index of bacterial wilt (resistant) with considerable additive effects and low dominant effects, suggesting that the inheritance of the bacterial wilt rate and index in tobacco mainly relies on additive inheritance.

Key words: Tobacco bacterial wilt; Major and minor locus groups; Genetic analysis; Bacterial wilt resistance in tobacco

\section{INTRODUCTION}

Tobacco (Nicotiana tabacum L.) is one of the most important cash crops, and China ranks first in the world in terms of its cultivation area and total yield. The development of high-quality tobacco is critical to tobacco growers and the cigarette industry. The tobacco leaf is the main harvested organ of this plant, and the types of tobacco diseases have gradually increased with the extension of continuous cropping years and changes in tobacco varieties. Tobacco bacterial wilt, caused by Ralstonia solanacearum, is one of the most destructive diseases in tobacco production; it was first reported in North Carolina, USA. By the end of the 1940s, tobacco bacterial wilt caused serious losses to agricultural production in the USA and Indonesia. Moreover, as a result of atmospheric circulation and human factors, it was reported in Japan, Australia, South Korea, and other countries (Chen et al., 1997). The local climatic characteristics of these regions favor spread of tobacco bacterial wilt, resulting in serious losses in the tobacco-planting areas of the Yangtze River Basin and Southern China. This infection can lead to destructive losses, and it is widespread in the tobacco-planting areas of Fujian, Hunan, Guangdong, Sichuan, and Guizhou. Continuous cropping is conducive to the occurrence of tobacco bacterial wilt, and the spread of the disease has become increasingly serious in Northern China in recent years (Kong, 2003).

Tobacco bacterial wilt is a typical vascular wilt disease that endangers plant roots, stems, and leaves. In serious cases, it can lead to the withering of an entire field of tobacco plants, which may lead to huge economic losses for farmers. Currently, tobacco bacterial wilt is mainly prevented and controlled by chemical means in agricultural production. However, once the plants are affected, the infection cannot be effectively controlled by chemical drugs. In addition, the application of chemical pesticides leads to the development of drug resistance, toxicity, and accumulation of pesticide residues, which cause environmental pollution. Changes in physiological races also exacerbate the difficulties associated with prevention. Therefore, no satisfactory results have been achieved regarding the prevention and control of tobacco bacterial wilt. Furthermore, the sources of resistance to tobacco bacterial wilt can be divided into several different levels. A few local varieties with low resistance are classified into Level 1, and screened varieties with moderate or lower resistance are classified into Level 2. However, these varieties cannot be restored by hybridization, and thus exhibit no practical value for hybridization with susceptible varieties using conventional breeding methods (Clayton and Smith, 1942; Clayton, 1948). Most genetic analyses indicate that different resistance sources possess various inheritance modes of resistance (Matsuda and Ohashi, 1973; Yang et al., 2005; Yang et al., 2006). Gai et al. (2003) proposed the mixed 
major gene plus polygene hypothesis of the quantitative trait genetic system, which suggests the following: 1) the number and effects of genes controlling quantitative traits are influenced by the environment; 2) genes with considerable effects that can be detected under normal experimental conditions are major genes, and those with lower effects that cannot be detected under existing experimental conditions are polygenes or minor genes; 3) major genes are relative to polygenes, and the genetic system of quantitative traits can be composed of only major genes, only polygenes, or a combination of major genes and polygenes. A quantitative trait genetic system composed of major genes and polygenes is known as a major gene plus polygene hybrid genetic system. The genetic patterns composed of pure major genes or pure polygenes (minor genes) are considered exceptions of the major gene plus polygene hybrid genetic system. In this study, the major and minor locus groups of bacterial wilt resistance in tobacco were analyzed for the first time, which provided references for the breeding of bacterial wilt-resistant tobacco.

\section{MATERIAL AND METHODS}

\section{Experimental materials}

Based on the tobacco germplasms identified by Sun et al. (2011a), different tobacco varieties with various levels of resistance to bacterial wilt were selected to construct the $F_{1}$ combinations of parental inbred lines and orthogonal diallel crosses in germplasms from 2009 (15 germplasms), 2010 (15 germplasms), and 2011 (16 germplasms). A total of 1/2P $(P+1)$ experimental materials were obtained.

The experimental tobacco germplasms were as follows: Yunyan 85 (P1), K326 (P2), LMAFC34 (P3), Coker86 (P4), Baisezhong (P5), RG11 (P6), DB101 (P7), NC86 (P8), K346 (P9), G80 (P10), Yanyan 97 (P11), Coker176 (P12), C17 (P13), Sheyuan 4 (P14), K399 (P15), and Changbohuang (P16). C17 is a self-bred line from the Tobacco Research Institute of the Anhui Academy of Agricultural Science.

\section{Field trial design}

A randomized block design with three replications was employed, and DB101 and Changbohuang were used as resistant and susceptible controls, respectively. Tobacco bacterial wilt incidences were identified in 2009, 2010, and 2011.

\section{Identification of disease resistance}

All experimental materials were cultivated in the bacterial wilt disease nursery of the Tobacco Research Institute of the Anhui Academy of Agricultural Science in Yixing Village, Hanting Town, Xuancheng City, Anhui Province. In accordance with the methods described in High density identification method (Sun et al., 2011b), tobacco plants were cultivated in flat furrows with a high density of 4444 plants $/ 667 \mathrm{~m}^{2}$ and 32 plants per plot (Sun et al., 2011c).

\section{Disease survey}

The incidence of tobacco bacterial wilt was surveyed on the 15th and 25th days during the early stage in accordance with the investigation method (Zhu et al., 1996). 


\section{Data analysis and construction of genetic models}

Data analysis and identification were conducted using the SAS8.1 software (SAS Institute Inc., 1997). The effects of the major and minor loci were analyzed based on the major and minor locus group inheritance model proposed by He et al. (2010). Using the major gene plus polygene inheritance model, the effect $\left(\mathrm{C}_{\mathrm{i} k}\right)$ of parents $\mathrm{i}$ and $\mathrm{k}$ can be decomposed into the sum of the major locus group effect $\left(G_{i k}\right)$ and the minor locus group effect $\left(W_{i k}\right)$ :

$$
C_{i k}=G_{i k}+W_{i k}
$$

where

$$
\begin{aligned}
& \left.\mathrm{G}_{\mathrm{ik}}=\sum_{j}\left\{a J[f(i, J)+f(k, j)] / 2+d J[f(i, J)+f(k, J)]^{2} / 4\right]\right\} \quad \text { (Equation 2) }
\end{aligned}
$$

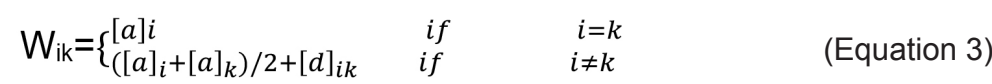

$J$ indicates the type of genetic variation of major locus groups; $a$ and $b$ indicate the additive and dominant effects of major locus groups, respectively; [a] and [d] indicate the total additive and dominant effects of minor locus groups, respectively; and $f(i, J)$ indicates the function of $i$ and $J$ that can be used to calculate the genotype based on the serial number of parents and the type of genetic variation. Specifically, $f$ is expressed as follows:

$$
f(I, J)=[-1]^{x}
$$

(Equation 4)

where $x=\operatorname{int}\left[(J-0.5) / 2^{n-i}\right] ; n$ indicates the total number of parents; and int indicates the bracket function. Thus, the linear model of interaction between the major and minor locus groups and the environment was constructed as follows:

$$
Y_{i k t l}=\mu+G_{i k}+E_{\mathrm{t}}+(G E)_{i k t}+B_{1(t)}+W_{i k}+(W E)_{i k t}+e_{i k t l} \quad \text { (Equation 5) }
$$

\section{RESULTS}

\section{Genetic analysis of major and minor locus groups based on incidence rate on the 15th day during the early stage}

Statistical analyses showed that major locus groups exhibited significant effects on the 
incidence rate on the 15th day during the early stage, whereas minor locus groups exhibited no significant effects. The variation derived from major and minor locus groups accounted for 23.96 and $13.05 \%$ of the phenotypic variation, respectively, and the broad heritability was $37.01 \%$.

Based on the experimental results, in the major locus groups $\mathrm{J} 1=13,055$ and $\mathrm{J} 2=3480$, the homozygous dominant locus $(++)$ increased the rate of tobacco bacterial wilt (susceptible), whereas the homozygous recessive loci (- -) were resistant. The genotypes of two major-minor locus groups in 15 parents and the derived combinations were divided into four types: parent P1 was classified as Type 1; parents P2, P3, P6, P9, P10, and P12 were classified as Type 2; parents $\mathrm{P} 8, \mathrm{P} 11, \mathrm{P} 13$, and P14 were classified as Type 3; parents P5, P7, and P15 were classified and Type 4. Among the parental genotypes, homozygous recessive loci that reduced the incidence rate (resistant) exhibited considerable additive effects. Specifically, the homozygous dominant loci and heterozygous loci of $\mathrm{J} 1=13,055$ (positive additive effect), which increased the incidence rate (susceptible), exhibited considerable additive and dominant effects. However, the homozygous dominant loci and heterozygous loci of $\mathrm{J} 1=3480$ (negative additive effect), which reduced the incidence rate (resistant), exhibited lower dominant effects.

The results indicated that the additive effects of minor locus groups on the rate of tobacco bacterial wilt in 15 parents varied from -24.7020 to 15.7012 . Furthermore, parent P15 exhibited the lowest additive effect and parent P7 exhibited the considerable additive effect. The dominant effects of minor locus groups on the rate of tobacco bacterial wilt in 105 combinations varied from -18.5895 to 35.0517 . Specifically, the P13 x P14 combination exhibited the lowest dominant effects and the P 6 x P10 combination exhibited the considerable dominant effects.

\section{Genetic analysis of major and minor locus groups based on incidence rate on the 25th day during the early stage}

Statistical analyses showed that major locus groups exhibited significant effects on the incidence rate on the 25th day during the early stage, whereas minor locus groups exhibited no significant effects. The variation derived from major and minor locus groups accounted for 10.40 and $10.23 \%$ of the phenotypic variation, respectively, and the broad heritability was $20.63 \%$. As shown in Table 1, the homozygous dominant loci of the major locus group $\mathrm{J} 1=671$ increased the rate of tobacco bacterial wilt (susceptible), whereas the homozygous recessive loci exhibited resistance. The genotypes of two major-minor locus groups in 15 parents and the associated combinations were divided into two types: parents P6, P11, P12, P13, and P14 were classified as Type 1; other parents were classified as Type 2 . These classifications suggested that among the parental genotypes, homozygous recessive loci, which reduced the incidence rate (resistant), exhibited considerable additive effects. In contrast, homozygous dominant loci and heterozygous loci (positive additive effect), which increased the incidence rate (susceptible), exhibited lower dominant effects.

As shown in Table 2, the additive effects of minor locus groups on the rate of tobacco bacterial wilt in 15 parents varied from -33.7212 to 14.3498 . Moreover, parent P18 exhibited the lowest additive effects, and parent P6 exhibited the considerable additive effects. The dominant effects of the minor locus groups on the rate of tobacco bacterial wilt in 105 combinations varied from -22.9167 to 29.4398 . Specifically, the P4 x P15 combination exhibited the lowest dominant effects, and the P8 x P14 combination exhibited the considerable dominant effects. 


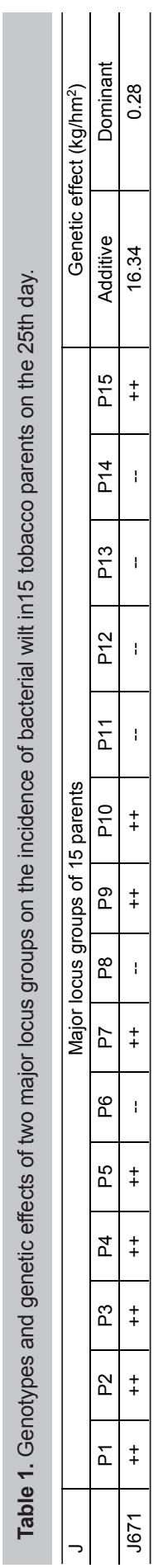




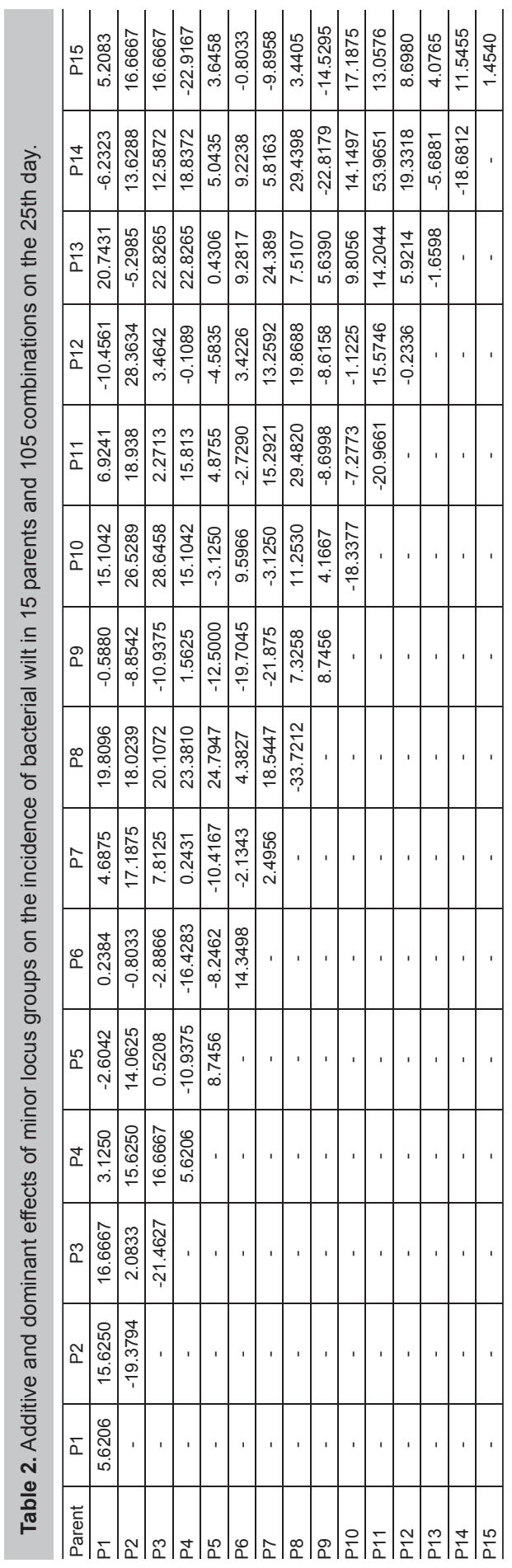




\section{Genetic analysis of major and minor locus groups based on the disease index on the 15th day during the early stage}

Statistical analyses indicated that the major locus groups exhibited significant effects on the disease index on the 15th day during the early stage, whereas minor locus groups exhibited no significant effects. The variation derived from major and minor locus groups accounted for 14.39 and $11.83 \%$ of the phenotypic variation, respectively, and the broad heritability was $26.22 \%$.

According to the experimental results, homozygous dominant loci in the major locus groups $\mathrm{J} 1=14,079$ and $\mathrm{J} 2=160$ were susceptible, whereas homozygous recessive loci were resistant. The genotypes of two major-minor locus groups in 15 parents and the associated combinations were divided into four types. Among the parental genotypes, homozygous recessive loci, which reduced the disease index (resistant), exhibited considerable additive effects, especially in the major locus group $\mathrm{J} 1=14,079$.

The results also suggested that the additive effects of minor locus groups on the tobacco bacterial wilt index in the 15 parents varied from -7.1479 to 15.665. Specifically, parent P8 exhibited the lowest additive effects, and parent P7 exhibited the considerable additive effects. The dominant effects of minor locus groups on the tobacco bacterial wilt index in the 105 combinations varied from -18.8400 to 21.6062 . Moreover, the P2 x P7 combination exhibited the lowest dominant effects, and the P10 x P15 combination exhibited the considerable dominant effects.

\section{Genetic analysis of major and minor locus groups based on the disease index on the 25th day during the early stage}

Statistical analyses showed that major locus groups exhibited significant effects on the disease index on the 25th day during the early stage, whereas minor locus groups exhibited no significant effects. The variation derived from major and minor locus groups accounted for 26.88 and $16.38 \%$ of the phenotypic variation, respectively, and the broad heritability was $43.25 \%$.

As shown in Table 3, homozygous dominant loci of major locus groups $\mathrm{J} 1=13,023$ and $\mathrm{J} 2$ $=1210$ increased the tobacco bacterial wilt index, whereas homozygous recessive loci exhibited resistance. The genotypes of two major-minor locus groups in the 15 parents and the associated combinations were divided into four types. Among the parental genotypes, homozygous recessive loci, which reduced the disease index, exhibited considerable additive effects, especially in the major locus group $\mathrm{J} 1=13,023$.

As shown in Table 4, the additive effects of minor locus groups on the tobacco bacterial wilt index in the 15 parents varied from -12.8012 to 19.4233 . Specifically, parent P14 exhibited the lowest additive effects, and parent P6 exhibited the considerable additive effects. The dominant effects of minor locus groups on the tobacco bacterial wilt index in the 105 combinations varied from -34.8856 to 36.9078 . Moreover, the P1 x P11 combination exhibited the lowest dominant effects, and the P17 x P13 combination exhibited the considerable dominant effects. 


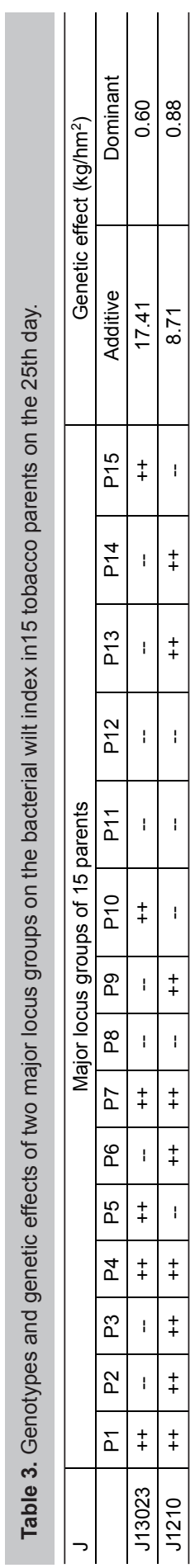




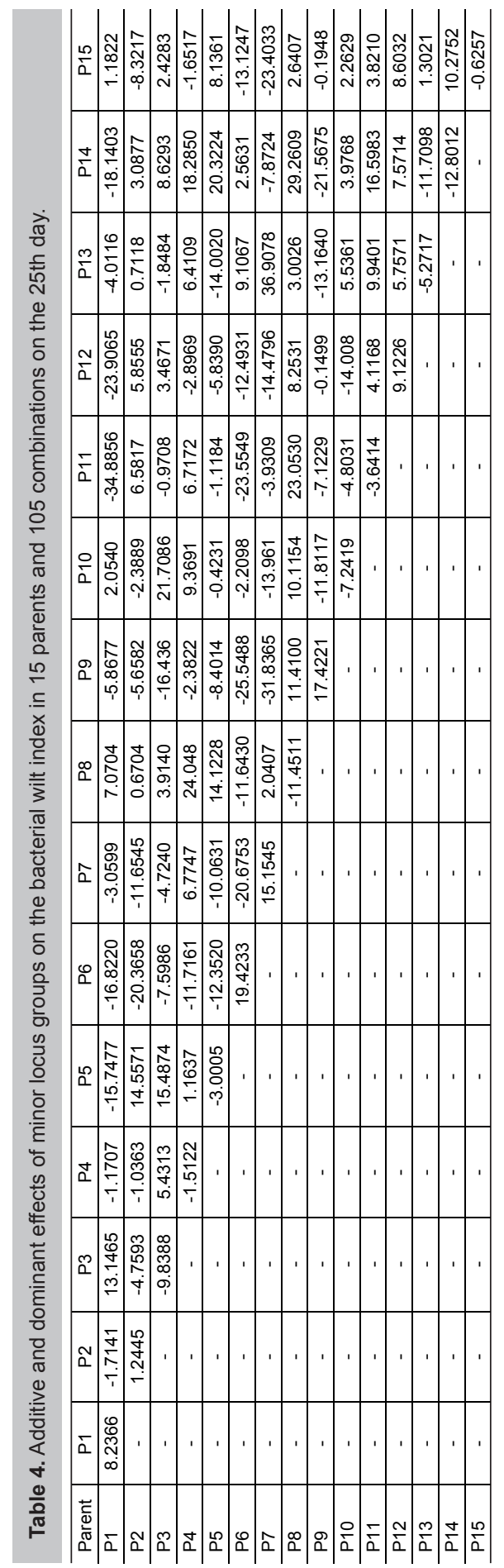




\section{DISCUSSION}

At present, no unified conclusion has been reached regarding the genetic mechanisms of bacterial wilt resistance in tobacco. The mixed major gene plus polygene hypothesis of plant quantitative traits proposed by Gai et al. (2003) has been used in the analyses of heterosis in several crops (Ma et al., 1983; Orf et al., 1999; Yang and Gai, 2009; Ni et al., 2011). Based on the major locus group analyses, the genetic structures and genetic effects of various major locus groups of different parents were demonstrated. These results not only clarified the genetic characteristics of various hybrid combinations, but they also provided the basis for further exploration of genetic potential. Parents can be purposefully designed and improved based on the genotypes and genetic effects of major locus groups, thus changing the unfavorable major locus groups into favorable ones. To improve the combinations of unfavorable major locus groups with synergistic genes provided by donor parents, the donor parents should be selected based on major locus group features. Matsuda and Ohashi (1973) reported that the inheritance of bacterial wilt resistance observed in several tobacco varieties was commonly controlled by single genes and polygenes. Using the major gene plus polygene analysis method, Wang et al. (2009) conducted genetic analyses on plant height traits, and they found that the results were in line with two pairs of additive-dominantepistatic major genes + additive-dominant polygenes. This result is based on the mixed inheritance model (E1), and it suggests that the quantitative trait separation analysis method could also be used to investigate other quantitative tobacco traits. Gao et al. (2010) found that the bacterial wilt resistance in tobacco was controlled by two pairs of additive-dominant-epistatic major genes + additive-dominant polygenes, and $\mathrm{Ni}$ et al. (2011) reported that the inheritance of the easy curing trait of tobacco leaves was in line with this rule. The results of this study are consistent with those of previous studies.

In the present study, the major and minor locus group analysis method was developed based on the major gene plus polygene inheritance model. The additive-dominant effects of majorminor locus group and its interaction with environment were used to analyze the genetic structures of bacterial wilt resistance in tobacco and the genetic effects of major and minor locus groups, thereby providing a reference for genetic improvements in bacterial wilt-resistant tobacco breeding. According to the results, major-minor locus groups exhibited significant effects on the rate and index of bacterial wilt, whereas minor locus groups exhibited no significant effects. Specifically, the effects on the incidence rate of Yunyan 85, DB101, and RG11 reached the maximum, and the effects on the disease index of the latter two germplasms also reached the maximum. The detection results were consistent with disease-resistance indicators of these three varieties in the field, and they were consistent with the corresponding broad heritability, which was >20 and $>26 \%$ for incidence rate and disease index, respectively. Genetic homozygous dominant loci (+ + ) of bacterial wilt resistance indicators increased the rate of bacterial wilt (susceptible). However, homozygous recessive loci (- -) exhibited resistance with considerable additive effects and low dominant effects, which indicated that the inheritance of bacterial wilt resistance in tobacco mainly relies on additive inheritance.

Based on the genetic effects of the major and minor locus groups on the rate and index of bacterial wilt in tobacco on the 15 and 25th day during the early stage, the major locus groups exhibited significant effects, but minor locus groups did not exhibit significant effects. The effects on the incidence rate of Yunyan 85, DB101, and RG11 reached the maximum, and the effects on the disease index of the latter two germplasms reached the maximum. These results were consistent with disease-resistance indicators of these tobacco varieties in the field, with corresponding broad 
heritability values $>20 \%$. Furthermore, the genetic resistance of Yunyan 85, DB101, and RG11 should be utilized for the improvement of bacterial wilt resistance in tobacco varieties. Genetic homozygous dominant loci $(++)$ of bacterial wilt resistance indicators increased the incidence rate (susceptible), whereas homozygous recessive loci (- -) reduced the disease index (resistant) with considerable additive effects and low dominant effects. These results suggested that the inheritance of the bacterial wilt rate and index in tobacco mainly relies on additive inheritance. Thus, the genetic overlap of additive effects can be realized by sexual hybridization in production practices.

\section{Conflicts of interest}

The authors declare no conflict of interest.

\section{ACKNOWLEDGMENTS}

We would like to thank the Anhui Provincial Corporation of China National Tobacco Corporation for the financial support of Tobacco high-quality-and-Resistance Pyramid Breeding and Bacterial-Wilt-Resistance Genes Analysis (Grant \#20100551003) and Identification System Study of Tobacco Bacterial Wilt Resistance Based on Molecular Markers (\#AHYCKJ2014032) for financial support of the project.

\section{REFERENCES}

Chen RT, Zhu XC, Wang ZF, Guo ZY, et al. (1997). A report of investigating and studying tobacco infectious diseases of 16 main tobacco producing provinces (regions) in China. Acta Tabacaria Sin 4: 1-7.

Clayton EE (1948). A wildfire resistant tobacco. J. Hered. 38: 35-40.

Clayton EE and Smith TE (1942). Resistance of tobacco to bacterial wilt (Bacterium solanacearum). J. Agric. Res. 65: 547-554.

Gai JY, Zhang YM and Wang JK (2003). Genetic system of quantitative traits in plants. Science Press, Beijing.

Gao JM, Wang ZD and Zhang WX (2010). Genetic analysis on resistance to bacterial wilt in oriental tobacco. Chin. Tob. Sci 31: 1-4.

He JB, Guan RZ and Gai JY (2010). A genetic analysis method of major-minor locus groups in diallel cross design. Acta Agron. Sin. 36: 1248-1257. http://dx.doi.org/10.3724/SP.J.1006.2010.01248

Kong FY (2003). Integrated control of tobacco bacterial wilt disease. Tob. Sci. Technol 4: 42-48.

Ma YH, Gai JY and Hu YZ (1983). Studies on genetic variation of successive generations after hybridization in soybeans: II. Combining ability and related genetic parameters. Acta Agron. Sin. 9: 249-258.

Matsuda T and Ohashi $Y$ (1973). Inheritance of resistance to bacterial wilt disease in tobacco: II. Inheritance of resistance originated from T.I. 448A and relation between those and Rps gene. Jpn. J. Breed 23: 175-180. http://dx.doi.org/10.1270/jsbbs1951.23.175

$\mathrm{Ni} \mathrm{C}, \mathrm{Xu}$ XH and Zhang XW (2011). Genetic analysis of easy curing potential in flue-cured tobacco with the mixed major-gene plus polygene inheritance model. Chin. Tob. Sci 32: 1-4.

Orf JH, Chase K, Jarvik T, Mansur LM, et al. (1999). Genetics of soybean agronomic traits: I. Comparison of three related recombinant inbred populations. Crop Sci. 39: 1642-1651. http://dx.doi.org/10.2135/cropsci1999.3961642x

SAS Institute Inc (1997). SAS/STAT software: changes and enhancements through release 6.12. SAS Institute Inc., Cary, North Carolina.

Sun XY, Zhou YB, Yang HY, Gao ZL, et al. (2011a). Identification and classification of tobacco germplasm resistant to bacterial wilt. Acta Tabacaria Sin 19: 61-66.

Sun XY, Zu CL, Gao ZL, et al. (2011b). High density identification method of bacterial wilt resistance in tobacco in the field. China National invention patent, authorized patent number: ZL200910144985.4

Sun XY, Zu CL and Gao ZL (2011c). Influence of high plant density on morphology characters and efficacy of resistant identification to bacterial wilt in tobacco. Acta Tabacaria Sin 17: 77-82.

Wang RX, Ren M, Zhang XW, Chang A, et al. (2009). Genetic analysis of plant height using mixed major gene plus polygenes inheritance model in culture spawn of Nicotiana tabacum L. Chin. Tob. Sci 30: 15-20. 
Yang JY and Gai JY (2009). Heterosis, combining ability and their genetic basis of yield among key parental materials of soybean in Huang-Huai Valleys. Acta Agron. Sin. 35: 620-630. http://dx.doi.org/10.3724/SP.J.1006.2009.00620

Yang YC, Zhou QM and Yin HQ (2005). Establishment and optimization of AFLP reaction system in tobacco. Acta Tabacaria $\sin 11: 23-28$.

Yang YC, Zhou QM and Zhu LS (2006). Heredity and RAPD markers analysis of resistance gene to tobacco bacterial wilt. Acta Tabacaria Sin 12: 38-42.

Zhu XC, Shi JK, Kong FY, Guo YF, et al. (1996). YC/T 39-1996, Grade and investigating method of tobacco disease. China Nat. Tobacco Corp. 